\title{
Neuropeptide Hierarchies and the Activation of Sequential Motor Behaviors in the Hawkmoth, Manduca sexta
}

\author{
Stephen C. Gammie and James W. Truman \\ Zoology Department, University of Washington, Seattle, Washington 98195-1800
}

In insects, the shedding of the old cuticle at the end of a molt involves a stereotyped sequence of distinct behaviors. Our studies on the isolated nervous system of Manduca sexta show that the peptides ecdysis-triggering hormone (ETH) and crustacean cardioactive peptide (CCAP) elicit the first two motor behaviors, the pre-ecdysis and ecdysis behaviors, respectively. Exposing isolated abdominal ganglia to ETH resulted in the generation of sustained pre-ecdysis bursts. By contrast, exposing the entire isolated CNS to ETH resulted in the sequential appearance of pre-ecdysis and ecdysis motor outputs. Previous research has shown that ETH activates neurons within the brain that then release eclosion hormone within the CNS. The latter elevates cGMP levels within and increases the excitability of a group of neurons containing CCAP. In our experiments, the ETH-induced onset of ecdysis bursts was always associated with a rise in intracellular cGMP within these CCAP neurons. We also found that CCAP immunoreactivity decreases centrally during normal ecdysis. Isolated, desheathed abdominal ganglia responded to CCAP by generating rhythmical ecdysis bursts. These ecdysis motor bursts persisted as long as CCAP was present and could be reinduced by successive application of the peptide. CCAP exposure also actively terminated preecdysis bursts from the abdominal CNS, even in the continued presence of ETH. Thus, the sequential performance of the two behaviors arises from one modulator activating the first behavior and also initiating the release of the second modulator. The second modulator then turns off the first behavior while activating the second.

Key words: CCAP; ecdysis; pre-ecdysis; ecdysis triggering hormone; eclosion hormone; cGMP
Neuromodulators (such as monoamines and neuropeptides) activate, alter, and create behavioral motor outputs from the nervous system. A major research question has been how these neuromodulators produce their distinct motor outputs. The most detailed work regarding this question has examined the stomatogastric ganglion of decapod crustaceans. In the absence of modulators, this ganglion shows a basal level of activity (Moulins and Cournil, 1982). Diverse neuromodulatory inputs, however, cause the emergence of functional neural circuits that generate discrete behaviors by altering the cellular (e.g., intrinsic excitability) (Flamm and Harris-Warrick, 1986) and synaptic properties (e.g., increased strength of electrical coupling) (Johnson et al., 1993) of neurons within the ganglion (Simmers et al., 1995). The effect of neuromodulators on behavioral motor programs is also evident at the level of the whole animal. For example, octopamine activates flight in insects (Sombati and Hoyle, 1984), dopamine triggers walking in decerebrate cats (Grillner and Zangger, 1979), and serotonin elicits swimming in nudibranchs (McClellan et al., 1994) and aggressive posturing in lobsters (Kravitz, 1988).

Many behaviors, however, do not occur in isolation but occur as part of a behavioral sequence with distinct phases. For example, the mating behavior of most animals involves distinct precopulatory, copulatory, and postcopulatory behaviors that occur sequen-

Received Jan. 15, 1997; revised March 5, 1997; accepted March 10, 1997.

This work was supported by a predoctoral National Research Service Award Traineeship T32 GM-07270-21 to S.C.G. and National Science Foundation Grant IBN9511077 to J.W.T. We thank Dr. Nathan Tublitz for his generous gift of synthetic CCAP peptide and Dr. Jan de Vente for his generous gift of the anti-cGMP antibody. We also thank Nat Scholz for comments on this manuscript.

Correspondence should be addressed to Stephen C. Gammie, University of Washington, Seattle, WA 98195-1800.

Copyright (C) 1997 Society for Neuroscience $\quad 0270-6474 / 97 / 174389-09 \$ 05.00 / 0$ tially. If individual neuromodulators configure distinct motor behaviors, then an important question is what controls the release of the neuromodulators and how do the different modulators interact to produce a behavioral sequence.

A well documented behavioral sequence in insects is the series of behaviors used to shed the old cuticle (Carlson, 1977; Reynolds, 1980). In larvae of the moth Manduca sexta, the first phase, the pre-ecdysis behavior, involves rhythmic compression and relaxation movements along the length of the body that loosen the old cuticle (Copenhaver and Truman, 1982; Miles and Weeks, 1991). Ecdysis, the second phase, follows immediately and accomplishes the actual shedding of the cuticle via anteriorly directed peristaltic contractions (Weeks and Truman, 1984). This behavioral sequence was believed to be orchestrated by a single neuropeptide, eclosion hormone (EH) (Copenhaver and Truman, 1982), but recent studies have shown that a second peptide, ecdysistriggering hormone (ETH), is also involved (Zitnan et al., 1996). These two peptides stimulate the release of one another through a positive feedback loop, but neither peptide seems to be the final effector of the ecdysis motor output (Ewer et al., 1997).

$\mathrm{EH}$ release within the CNS causes cGMP upregulation within a set of neurons (the Cell 27/704 group) that are immunopositive for crustacean cardioactive peptide (CCAP) (Ewer et al., 1997). cGMP upregulation, which occurs just before ecdysis (Ewer et al., 1994), increases the excitability of these cells (Gammie and Truman, 1997). Here we show that CCAP acts centrally to cause the ecdysis motor output. We find that ETH and CCAP act in sequence to generate the first and second motor behaviors of the ecdysis sequence, respectively. The hierarchical response of the CNS to these peptides also facilitates the sequential appearance of motor behaviors. 


\section{MATERIALS AND METHODS}

Isolated nervous system preparation. Larvae of the tobacco hornworm Manduca sexta were raised at $26^{\circ} \mathrm{C}$ on an artificial diet (Bell and Joachim, 1976). Larvae molting from the fourth to the fifth instar were staged relative to ecdysis using external morphological markers (Copenhaver and Truman, 1982). Animals were anesthetized briefly on ice, decapitated, cut longitudinally along the dorsal midline, pinned out in a Sylgard (Dow Corning, Midland, MI)-coated dish, and bathed in a CNS saline containing (in $\mathrm{mM}$ ): $140 \mathrm{NaCl}, 5 \mathrm{KCl}, 4 \mathrm{CaCl}_{2}, 28 \mathrm{D}$-glucose, and 5 HEPES, adjusted to pH 7.4 using $\mathrm{NaOH}$ (Trimmer and Weeks, 1989). Typically, the ventral nerve cord from the second abdominal ganglion (A2) through the terminal ganglion (including the anterior and lateral roots of the A3 and A4 dorsal nerves) was dissected out and placed in a CNS saline containing 4\% collagenase/dispase (Boehringer Mannheim, Indianapolis, IN) for $15 \mathrm{~min}$ at $26^{\circ} \mathrm{C}$. The ganglia were then rinsed, and the ganglionic sheath on the dorsal and ventral surfaces of $\mathrm{A} 2, \mathrm{~A} 3$, and A4 was removed using fine forceps.

Extracellular recordings. Glass suction electrodes were used to record from either the anterior or lateral branches of the dorsal nerve from abdominal ganglia A3 and A4. The signals were amplified by a differential amplifier (Tektronix, Beaverton, OR) and sent to videotape through a VCR (A. R. Vetter, Rebersberg, PA) and a chart recorder (Gould Inc., Cleveland, $\mathrm{OH}$ ). The stored data were played back and analyzed on a Macintosh computer using Superscope (GW Instruments, Somerville, MA). All experiments were performed at room temperature.

Peptide treatments. Synthetic CCAP was a kind gift of Dr. Nathan Tublitz (University of Oregon). A stock solution of $10 \mu \mathrm{M}$ [kept frozen with $0.5 \%$ bovine serum albumin (Sigma, St. Louis, MO)] was used for dilutions. ETH (Zitnan et al., 1996) was synthesized by the Howard Hughes Macromolecular Synthesis unit at the University of Washington. A $1 \mathrm{~mm}$ stock was prepared in saline and frozen until it was diluted for experiments. In pilot experiments CCAP was added to the CNS saline just before the ganglia were desheathed, but in all subsequent experiments CCAP was added after the extracellular recordings had begun. The concentrations of CCAP that were used ranged from $10^{-10}$ to $10^{-6} \mathrm{M}$. The concentration of ETH that was used was always $10^{-6} \mathrm{M}$. For the experiment of washing out CCAP, the extracellular electrodes were removed temporarily, and the CNS was washed repeatedly with $1 \mathrm{ml}$ vol of CNS saline for $20 \mathrm{~min}$, after which the electrodes were replaced. For most experiments the CNSs were maintained in a volume of $1 \mathrm{ml}$ during the recording.

Immunocytochemistry. After each experiment, ganglia were placed in $4 \%$ paraformaldehyde in PBS overnight at $4{ }^{\circ} \mathrm{C}$, rinsed with $0.3 \%$ Triton $\mathrm{X}-100$ (Sigma) in PBS (PBS-X), and blocked with 5\% normal donkey serum (Jackson ImmunoResearch Laboratories, West Grove, PA) for 30 min before incubation overnight with primary antibodies. A sheep anticGMP antiserum (a kind gift of Dr. Jan de Vente) was used at a concentration of 1:20,000. The tissue was washed with PBS-X and placed overnight in a PBS-X solution containing a peroxidase-conjugated donkey anti-sheep IgG (1:200 dilution; Jackson Labs). After rinses in PBS-X, tissues were incubated in a $0.5 \mathrm{mg} / \mathrm{ml}$ diaminobenzidine (Sigma) PBS-X solution with $0.003 \% \mathrm{H}_{2} \mathrm{O}_{2}$ to form a brown precipitate. Ganglia were dehydrated, cleared in xylene, and mounted in DPX (Fluka, Buchs, Switzerland).

Immunostaining for CCAP used a rabbit anti-CCAP antiserum from Dr. H. Agricola (University of Jena). Molting larvae at defined times during ecdysis to the second larval stage were opened along the dorsal midline, pinned out flat, and fixed in $4 \%$ formaldehyde in PBS. After they were washed in PBS-X, tissues were then exposed to 1:1000 anti-CCAP antiserum in PBS-X and 1\% normal donkey serum for $36 \mathrm{hr}$. After repeated washings, tissues were then incubated overnight with a 1:1000 dilution of FITC-conjugated donkey anti-rabbit IgG (Jackson Labs). After repeated washings, the preparations were dehydrated through an ethanol series, cleared in xylene, and mounted in DPX.

For confocal analysis of CCAP release, nervous systems from larvae in the various treatment groups were processed together using immunocytochemical techniques. The preparations were then analyzed by confocal microscopy (Bio-Rad MRC 600; Bio-Rad, Hercules, CA). Data from all treated and control ganglia were collected during the same session using the same gain and black level settings. Horizontal optical sections were taken at $3 \mu \mathrm{m}$ steps through each ganglion, and the resultant z-series was then collapsed as a flat image using the "most intense pixel" algorithm. The collapsed image was then analyzed using National Institutes of Health Image. For each "collapsed" ganglion, the boundary between the cortex and neuropil was outlined, and the average intensity of fluores- cence was measured within that area. Background levels were measured from an adjacent region of ganglion cortex. The amount of CCAP-related fluorescence was then calculated as the neuropilar intensity minus background.

\section{RESULTS \\ CCAP activation of the ecdysis motor program}

In Manduca sexta, a distributed group of CCAP-containing neurons, the Cell 27/704 group, shows a dramatic increase in cGMP just before the onset of the ecdysis behavior (Ewer and Truman, 1994; Ewer et al., 1997). The appearance of cGMP in these CCAP neurons is a feature of ecdysis seen in a wide variety of insects (Ewer and Truman, 1996). In Manduca the cGMP rise increases the excitability of these neurons (Gammie and Truman, 1997), which raises the possibility that CCAP release might be directly related to the onset of ecdysis. CCAP has been isolated from Manduca and was initially named $\mathrm{CAP}_{2 \mathrm{~A}}$ (Cheung et al., 1992). We examined the effects of CCAP on a partially desheathed, isolated CNS consisting of the chain of abdominal ganglia (Fig. $1 D)$. These nervous systems also had their tracheal supply removed. We monitored motoneuron output by recording extracellularly from dorsal nerve roots (either the anterior or lateral branch) of abdominal ganglia A3 and A4, because these nerve roots contain axons of motoneurons known to be involved in ecdysis (Weeks and Truman, 1984). Most recordings were taken from the anterior branch, because this branch contains the axons of only two motoneurons, MN-2 and MN-3, and these motoneurons participate in both pre-ecdysis and ecdysis (Weeks and Truman, 1984; Novicki and Weeks, 1995).

Nervous systems were taken from larvae before the appearance of air in the old head capsule, at least $5 \mathrm{hr}$ before normal ecdysis (Copenhaver and Truman, 1982). Spontaneous activity consisted of low levels of tonic activity, with occasional unpatterned bursts. The addition of $10^{-8} \mathrm{M}$ CCAP to the isolated abdominal CNS induced rhythmic patterned bursting within 2-10 min (mean = $5.1 \pm 1.1 \mathrm{~min} ; \pm \operatorname{SEM}, n=8$; Fig. $1 A$ ). The bursts occurred with an average cycle period of $18.7 \pm 0.6 \mathrm{sec}( \pm \mathrm{SEM} ; n=12)$ and with a range from 15 to $22 \mathrm{sec}$. The bursts also showed an anterior progression up the chain of ganglia with a phase delay between segments of approximately one quarter of a cycle period (Fig. $1 B$ ). This CCAP-induced pattern of motor activity was similar to that recorded from deafferented nervous systems of Manduca during larval ecdysis (Weeks and Truman, 1984). The latter showed a cycle period between 24 and $34 \mathrm{sec}$ and a phase delay between successive ganglia of 0.25 of the cycle period. Similar values for the ecdysis motor pattern were also seen by others (Zitnan et al., 1996) and by us after entire isolated CNS preparations were exposed to ETH. Our preparations, for example, showed an average cycle period of $18.2 \pm 0.7 \sec ( \pm \mathrm{SEM} ; n=4)$. Although also metachronous, the pilocarpine-inducible crawling motor output of Manduca is dissimilar from the ecdysis motor program because the anterior nerve root does not show activity during crawling (Johnston and Levine, 1996).

As seen in Figure $1 B$, the ecdysis motor pattern was stable in the continuing presence of CCAP. We had no instance in which the ecdysis program terminated spontaneously in the constant presence of CCAP $\left(10^{-9}-10^{-6} \mathrm{M} ; n=12\right)$. Through our longest recordings, which lasted $90 \mathrm{~min}$ (Fig. $1 \mathrm{~B}$ ), there was little change in either the cycle period or the phase relationship seen during the ecdysis motor bursts.

CCAP addition was effective only on desheathed nervous systems. As seen in Figure $1 C$, application of CCAP $\left(10^{-6} \mathrm{M}\right)$ to 

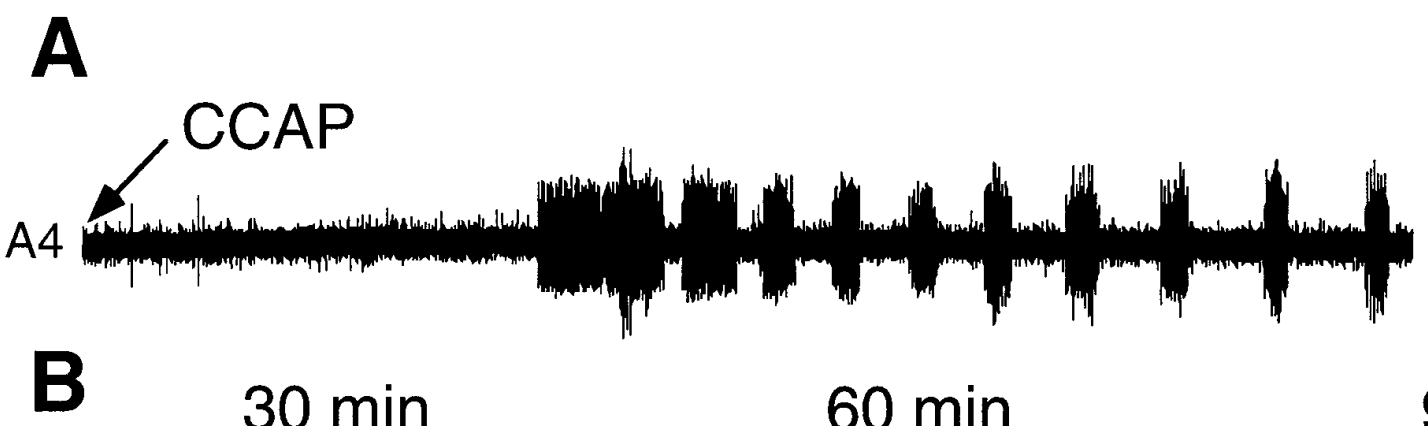

\section{$30 \min$}
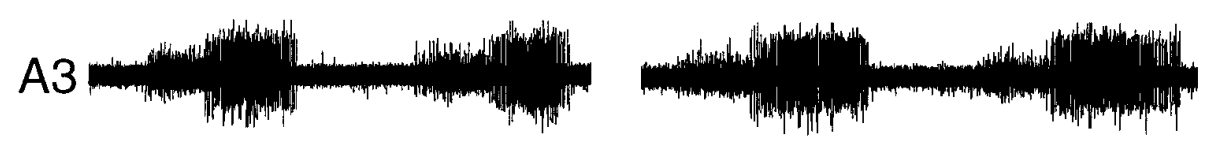

$60 \min$

\section{$90 \min$}
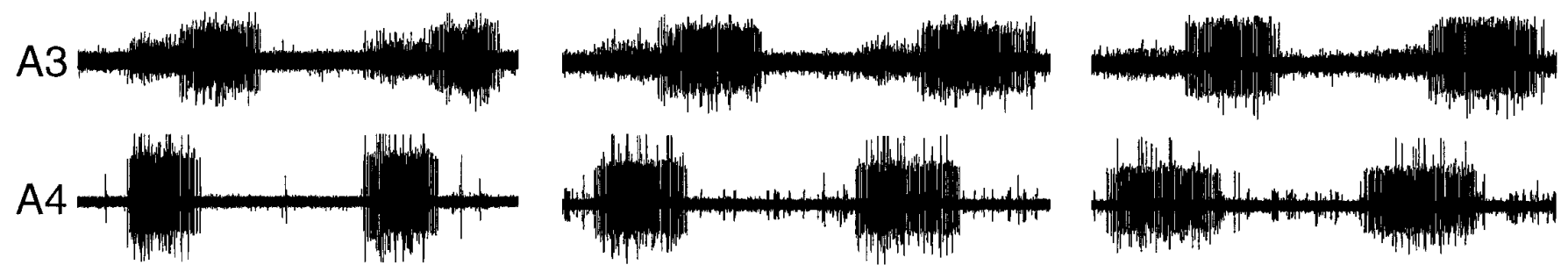

C

60 min (Sheath intact + CCAP)

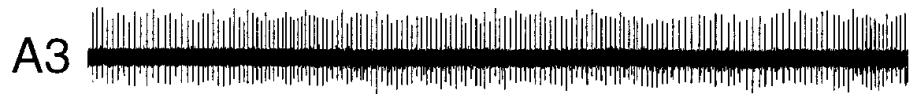
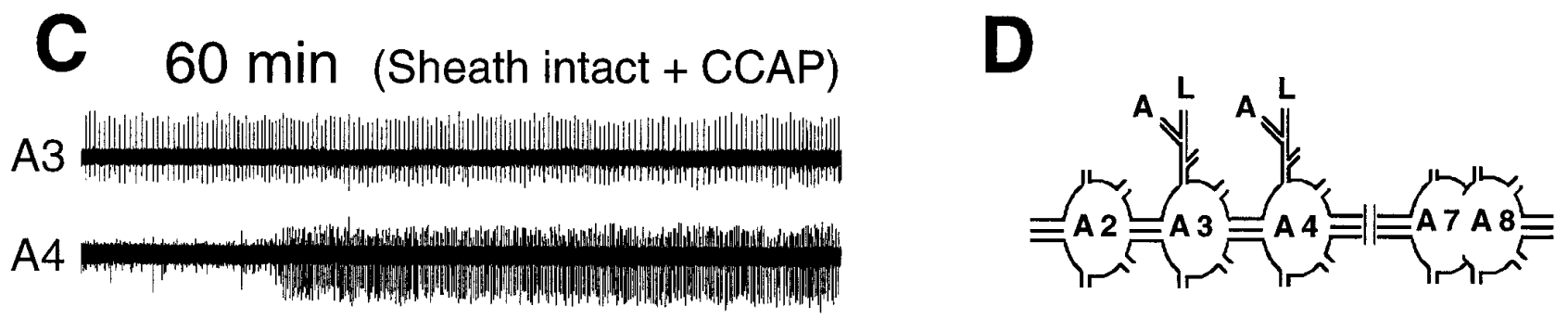

Figure 1. Extracellular recordings showing the effect of CCAP on the isolated abdominal CNS. $A$, Low-speed record showing the onset of rhythmical motor bursts $\sim 2 \mathrm{~min}$ after application of $1 \mu \mathrm{M}$ CCAP to a desheathed preparation. $B$, Examples of records from a desheathed abdominal CNS at 30 , 60 , and $90 \mathrm{~min}$ after exposure to $0.1 \mu \mathrm{M}$ CCAP. The cycle period and phase delay were stable through the entire recording period. $C$, Extracellular recording from an abdominal CNS with the ganglionic sheath intact $60 \mathrm{~min}$ after addition of $1 \mu \mathrm{M}$ CCAP. CCAP elicited no rhythmical bursting from preparations with intact ganglionic sheaths. $D$, Schematic drawing of the abdominal CNS preparation used in most experiments. All recordings were taken from the anterior nerve roots of $\mathrm{A} 3$ and $\mathrm{A} 4$, unless stated otherwise. Calibration: $A, 14 \mathrm{sec} ; B, C, 5 \mathrm{sec}$.

isolated abdominal ganglia with an intact ganglionic sheath did not result in the induction of ecdysis motor bursts $(n=3)$. By contrast, with desheathed preparations we found that ecdysis bursts could be initiated with concentrations of CCAP as low as $10^{-9} \mathrm{M}$, but not as low as $10^{-10} \mathrm{M}$. In the range of CCAP concentrations that elicited ecdysis motor bursts $\left(10^{-9}\right.$ to $\left.10^{-6} \mathrm{M}\right)$, we saw no consistent effect of peptide concentration on burst frequency.

The effects of CCAP in inducing the ecdysis bursts were reversible. As seen in Figure 2, CCAP addition to the isolated abdominal CNS triggered the motor program, but after the CCAP was washed out, the motor program stopped. When we reapplied CCAP, the ecdysis output resumed (Fig. 2). The reversibility of CCAP action was observed in four of four such washout experiments.

\section{Effects of ETH on the isolated CNS}

When injected into intact molting larvae, ETH causes pre-ecdysis behavior followed by ecdysis (Zitnan et al., 1996). When injected into larvae that have their nerve cord transected below the brain, however, ETH evokes only the pre-ecdysis motor program posterior to the transection (Ewer et al., 1997). We compared the effect of ETH on the whole isolated CNS with its effect on the desheathed abdominal CNS to determine whether ETH could directly evoke the ecdysis motor program. For the abdominal
CNS, the addition of $1 \mu \mathrm{M}$ ETH to desheathed preparations elicited the onset of rhythmical motor bursts after an average delay of $8.4 \pm 1.1 \mathrm{~min}( \pm \mathrm{SEM} ; n=6)$ (Fig. $3 A)$. Unlike the bursts elicited by CCAP, these motor bursts were characterized by synchronized bursting of MN-2 and MN-3 from ganglia along the length of the abdominal chain. The motor bursts of the preecdysis behavior have been characterized extensively by Miles and Weeks (1991) and Novicki and Weeks (1995). The period between bursts ranges from 7 to $15 \mathrm{sec}$, and the duration of the bursts ranges from 5 to $9 \mathrm{sec}$. The axons of $\mathrm{MN}-2$ and $\mathrm{MN}-3$ reside in the anterior branch of the dorsal nerve, and consequently we recorded motor activity from this branch. The bursts elicited from the abdominal CNS by ETH had the frequency and patterning characteristic of pre-ecdysis behavior. They occurred with a periodicity of $7.5 \pm 0.2 \mathrm{sec}( \pm \mathrm{SEM} ; n=7)$. Successive ganglia showed synchronous bursting rather than the metachronous activity characteristic of ecdysis. As seen in Figure $3 B$, with continuous exposure to ETH, the abdominal CNS produced pre-ecdysis bursts for up to $90 \mathrm{~min}$ (the duration of the experiment). After $60 \mathrm{~min}$, however, the background firing between bursts tended to increase. ETH was able to trigger pre-ecdysis from the abdominal CNS both when the ganglionic sheath was intact and when it was removed. In no experiments involving ETH treatment of the isolated abdominal ganglia did we see ecdysis motor bursts after the pre-ecdysis bursts $(0 / 9)$. 
A
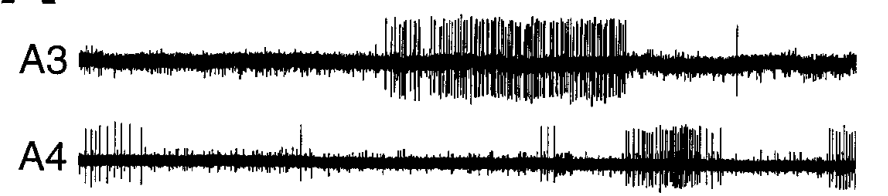

B

\section{+ CCAP}
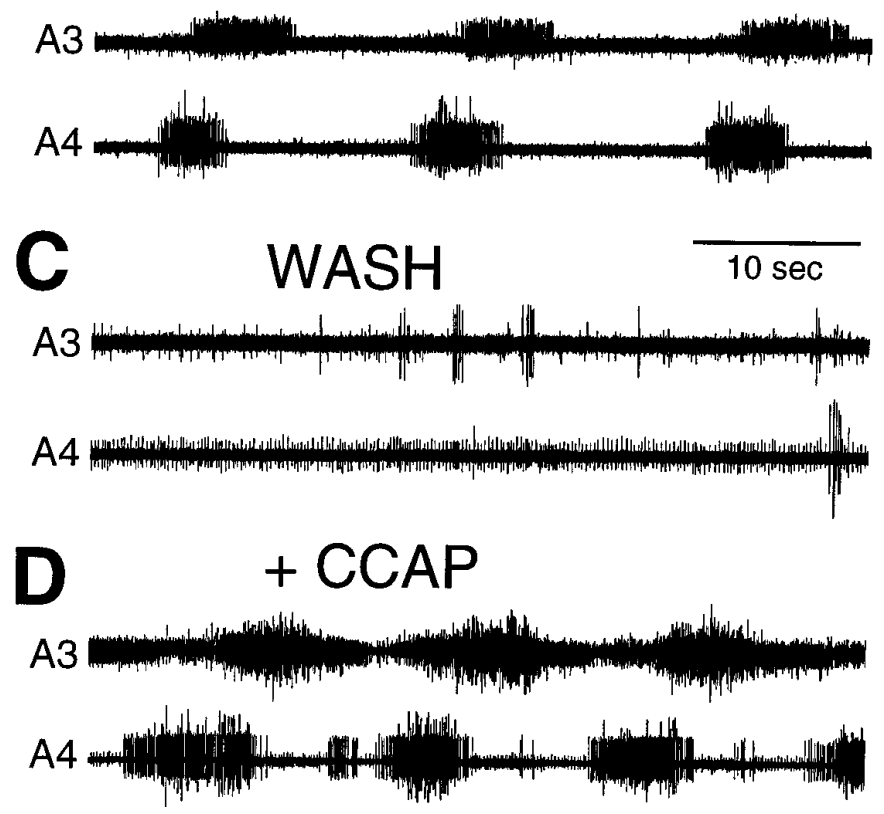

Figure 2. Extracellular recordings from a desheathed abdominal CNS taken $5 \mathrm{hr}$ before normal ecdysis showing the reversibility of CCAP action. $A$, Spontaneous motor activity before CCAP addition. $B$, Metachronous motor bursts characteristic of ecdysis occurred $10 \mathrm{~min}$ after application of $1 \mu \mathrm{M}$ CCAP; $C, 20$ min after CCAP washout. $D$, Ecdysis burst occurred again $5 \mathrm{~min}$ after reapplication of $1 \mu \mathrm{M}$ CCAP to the CNS. Electrodes were removed during the washout procedure between $B$ and $C$. Recordings in $C$ and $D$ were taken from the lateral nerve roots.

In contrast to our findings for the abdominal ganglia, Zitnan et al. (1996) reported that the entire isolated CNS responds to ETH by performing both the pre-ecdysis and ecdysis motor programs. As seen in Figure $4 A$, the same concentration of ETH $(1 \mu \mathrm{M})$ that caused only pre-ecdysis in the abdominal ganglia resulted in pre-ecdysis followed by ecdysis bursts in the entire isolated CNS. On average, pre-ecdysis motor bursts occurred for $41 \pm 4.5 \mathrm{~min}$ ( \pm SEM; $n=3$ ) before the onset of the ecdysis bursts. The transition from pre-ecdysis to ecdysis occurred over four to five cycles, with a set of bursts of uncertain patterning, possibly including a brief overlap of the two motor outputs (Fig. $4 A$ ). Thus, although the abdominal CNS is capable of generating ecdysis bursts, ETH can cause this response only if the abdominal ganglia are connected to the anterior portion of the CNS.

\section{Dominant effect of CCAP on the CNS}

The abdominal CNS generates two discrete motor programs, the pre-ecdysis and ecdysis motor programs in response to ETH and CCAP, respectively. To determine how these peptides might interact, we first exposed a desheathed abdominal CNS to $1 \mu \mathrm{M}$ $\mathrm{ETH}$, and minutes after the onset of pre-ecdysis bursts we added 100-fold less CCAP (10 nM). Ecdysis bursts first appeared approximately $10 \mathrm{~min}$ after CCAP was added, but during approximately the next 10 min we observed an intermingling of bursts having a pre-ecdysis character among the ecdysis bursts (Fig. 5). After this transition, however, only ecdysis bursts occurred, despite the continuing presence of ETH. The average duration of ETHinduced pre-ecdysis bursts in these experiments was only $29.7 \pm$ $1.7 \mathrm{~min}( \pm \mathrm{SEM} ; n=4)$. This duration represents the time from the onset of pre-ecdysis bursts through to the last pre-ecdysis burst during the overlap with ecdysis bursts. In contrast, the average duration of ETH-induced pre-ecdysis bursts when no CCAP was subsequently applied was $81.6 \pm 4.2 \mathrm{~min}( \pm \mathrm{SEM} ; n=5)$. The difference between these means are statistically significant (unpaired Student's $t$ test; $p<0.0001$ ). Consequently, the termination of pre-ecdysis bursts in the presence of CCAP likely did not result from the rundown of ETH action. In reciprocal experiments, we first added CCAP $(1 \mu \mathrm{M})$, and after the onset of ecdysis bursts we added ETH $(1 \mu \mathrm{M})$. In these experiments, however, ecdysis bursts continued undisturbed after ETH addition and only rarely did pre-ecdysis-like bursts appear, if at all (data not shown). Therefore, the CNS shows a hierarchical response to ETH and CCAP, and the peptide controlling the second behavior in the sequence has the ability to prematurely terminate the motor response caused by the earlier release of the first, even when the latter is still present.

\section{Developmental sensitivity of the CNS to CCAP}

Ecdysis behavior occurs only when an individual insect needs to shed its old cuticle. As a possible control mechanism, the sensitivity of animals to EH (Truman et al., 1983) or ETH (Zitnan et al., 1996) is confined to restricted phases of the molting period. We examined the sensitivity of the CNS to CCAP on the second day of the last larval instar, a time 2-3 d before the start of the pupal molt and when the animal responds to neither EH $(0 / 5)$ nor ETH (0/5). As seen in Figure 6, CCAP induced a robust ecdysis motor output from the abdominal CNS from this intermolt stage $(2 / 2)$. Thus, there seems to be no stage dependence on the ability of the CNS to respond to CCAP.

\section{cGMP immunoreactivity of the CNS}

The Cell 27/704 group of neurons contains CCAP (Davis et al., 1993; Ewer et al., 1994) and shows a striking increase in intracellular cGMP levels just before ecdysis (Ewer et al., 1994). Intracellular recordings from Cell 27 show that these cells increase their excitability because of this cGMP elevation (Gammie and Truman, 1997). Hence, the cGMP elevation in these cells is believed to be an important link in the chain of events leading to ecdysis (Ewer et al., 1997). Consequently, after the various experiments described above, we fixed the nervous systems in paraformaldehyde and assessed the levels of cGMP in the CNS using immunocytochemistry (De Vente et al., 1987). In cases in which the whole CNS was exposed to ETH, every CNS that entered into the ecdysis phase also showed cGMP immunoreactivity in the CCAP cells (Fig. 7). By contrast, when we examined the isolated abdominal CNSs that had been exposed to ETH (and that had only produced a pre-ecdysis output), we never detected cGMP in these cells (Fig. 7). Importantly, isolated abdominal CNSs that showed an ecdysis motor program in response to CCAP exposure also did not show the appearance of cGMP in the CCAP cells (Fig. 7). This last case is the only situation either in vivo or in the isolated CNS in which ecdysis is seen in the absence of the cGMP rise in the CCAP neurons. This result shows that the cGMP rise is not caused by the ecdysis motor program. Rather, it is consistent with the hypothesis that the cGMP elevation in these cells is in the chain of events leading to CCAP release and the subsequent ecdysis motor behavior. 


\section{A}

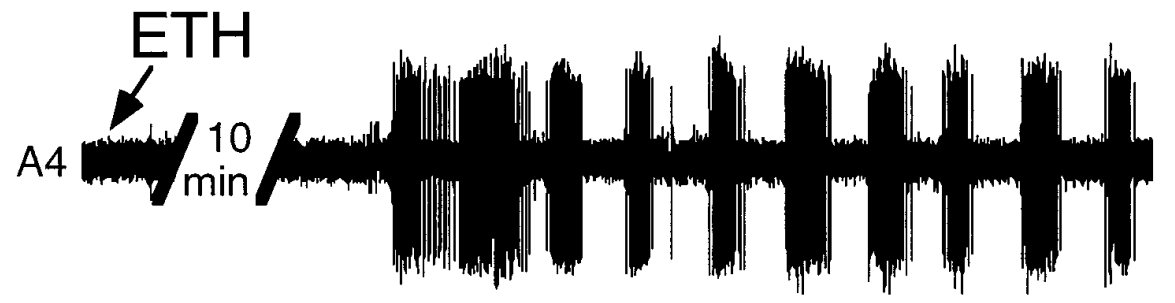

B

\section{$30 \min$}
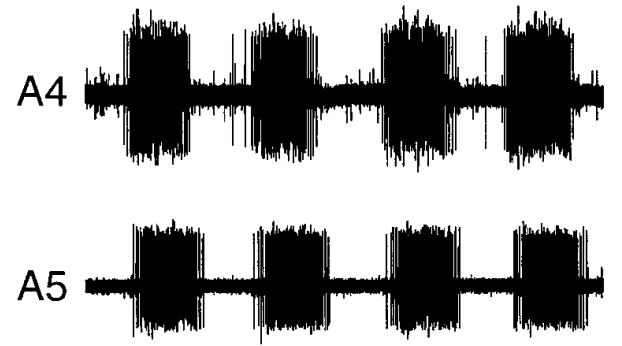

$60 \min$
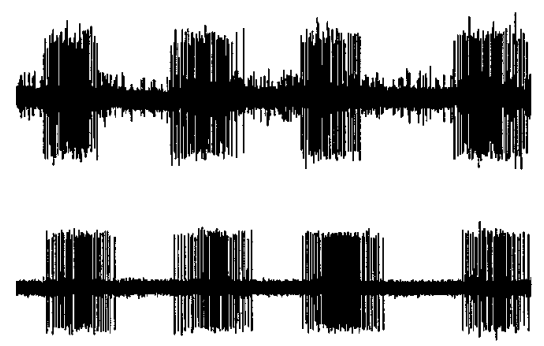

$90 \min$
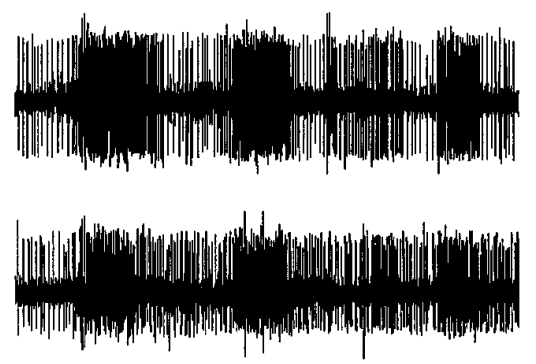

Figure 3. Extracellular recordings showing ETH action on the abdominal CNS. $A$, Low-speed record showing the onset of the pre-ecdysis motor bursts $\sim 10$ min after application of $1 \mu \mathrm{M}$ ETH. $B$, Extracellular recording 30, 60, and 90 min after application of $1 \mu \mathrm{M}$ ETH showing synchronous motor activity in the anterior nerve roots in successive segments. Pre-ecdysis bursts were still observable at 90 min. Calibration: $A, 11 \mathrm{sec} ; B, 7 \mathrm{sec}$.

A

\section{normal ecdysis}

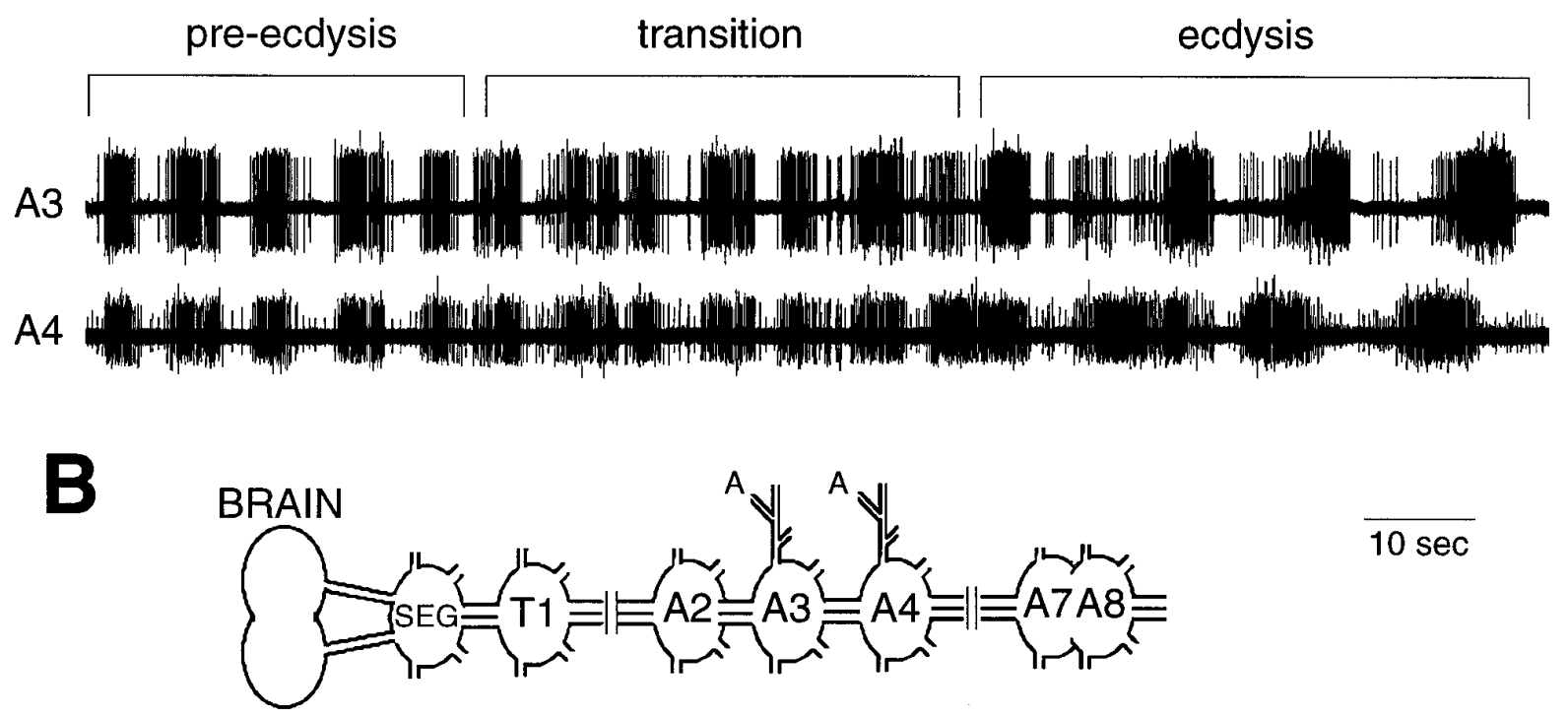

Figure 4. A, Extracellular recording from an isolated whole CNS exposed to $1 \mu \mathrm{M}$ ETH. The record shows the normal transition from pre-ecdysis to ecdysis bursts and was taken $60 \mathrm{~min}$ after ETH application. The transition was brief and may include some overlap of the two motor patterns. $B$, Schematic drawing of the whole CNS preparation showing the site of recording.

\section{Changes in CCAP immunoreactivity at ecdysis}

Larvae molting to the second instar were used to determine whether there was a measurable depletion of neuropilar CCAP around the time of ecdysis. We used CCAP immunocytochemistry along with confocal microscopy to estimate the CCAP content within the neuropil. For the larval ganglia, the neuropilar CCAP is caused by the arbors of the Cell 27 and 704 neuronal pairs. As seen in Figure 8, at the time that ecdysis behavior stopped, the average CCAP immunoreactivity in the ganglionic neuropil was about half of that seen before the start of pre-ecdysis.

For a group of larvae, a complete ring of old larval cuticle was removed at the level of A3. These "girdled" larvae could not shed 

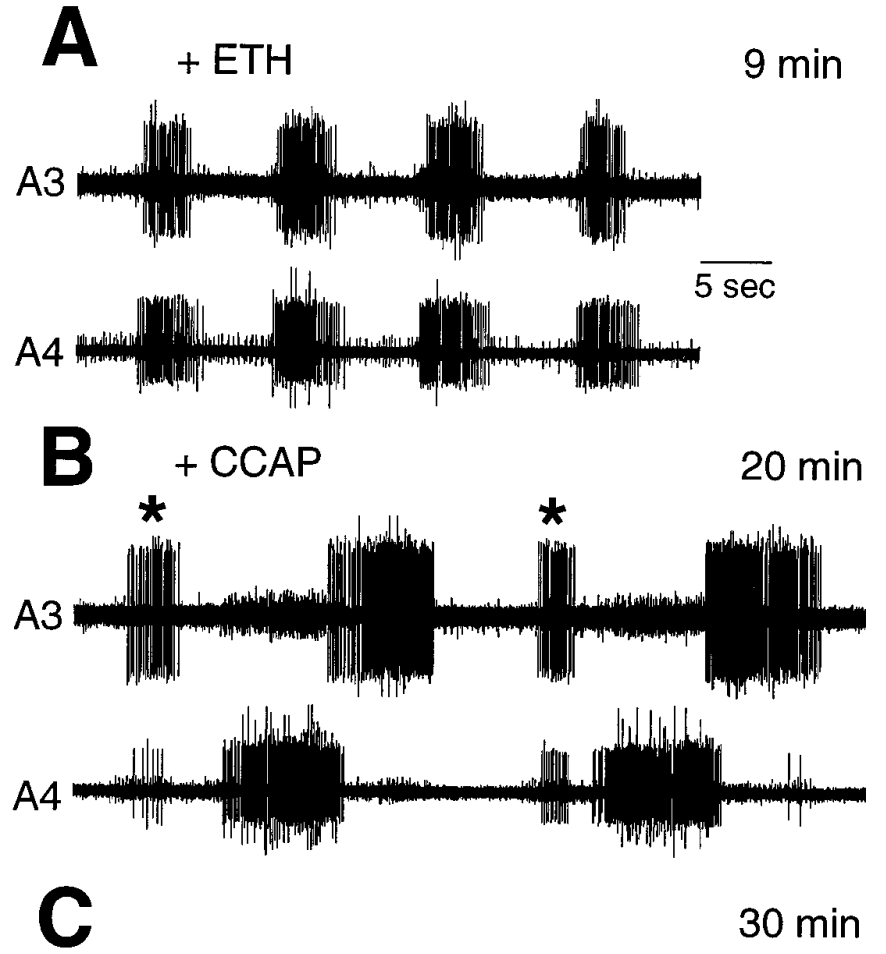

$30 \mathrm{~min}$

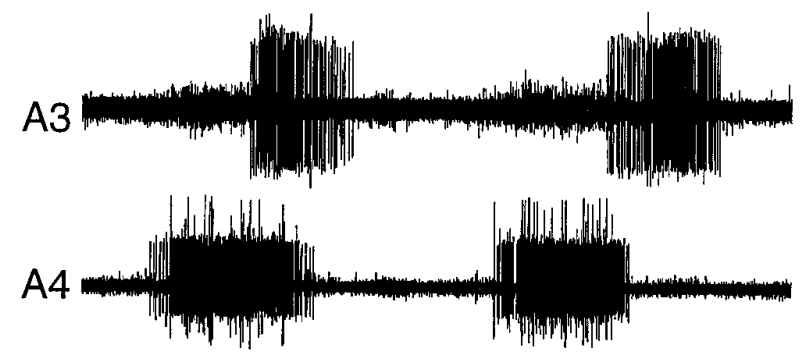

Figure 5. Extracellular recordings from the isolated abdominal CNS showing the dominant effect of CCAP over ETH. $A$, Recording 1 min after the onset of pre-ecdysis bursts elicited by the application of $1 \mu \mathrm{M} \mathrm{ETH} 8$ min earlier. $B$, Recording from the same preparation $10 \mathrm{~min}$ after addition of $0.1 \mu \mathrm{M}$ CCAP; pre-ecdysis bursts $\left(^{*}\right)$ were interspersed among the ecdysis bursts. CCAP was applied 2 min after the onset of the pre-ecdysis bursts. $C$, Ten minutes after $B$, only ecdysis bursts remain, although ETH is still present in the bath.

the old cuticle anterior to the ring, and they maintained ecdysis behavior for up to $1 \mathrm{hr}$ longer than unmanipulated animals. These larvae showed a more extreme loss of neuropilar CCAP as compared with normal larvae that had just finished ecdysis (Fig. 8A) or with age-matched controls that were 60-90 min after a normal ecdysis (data not shown). For example, in the ganglion in Figure $8 D$, the only significant stores of CCAP still remaining were in the cell bodies and the descending axon of Cell 27. This extreme depletion was evident in four of the five girdled animals.

\section{DISCUSSION}

Studies on decapod crustaceans have shown that in the absence of neuromodulators, the stomatogastric ganglion reverts to a quiescent state (Moulins and Cournil, 1982). Neuromodulators elicit behavioral outputs from this ganglion by altering the properties of neural circuits in different ways. Serotonin, for example, changes the intrinsic properties of cells within a circuit as well as the strength of synaptic interconnections (Flamm and HarrisWarrick, 1986; Johnson et al., 1993). In lobsters, it has also been

\section{+ CCAP}

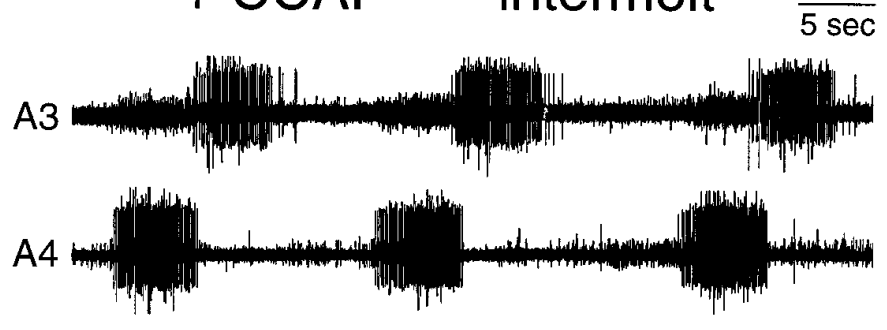

Figure 6. Extracellular record showing that CCAP action is not stage specific. The abdominal CNS of an intermolt animal on the second day of the fifth larval instar was desheathed and exposed to $1 \mu \mathrm{M}$ CCAP. Ecdysis bursts began within 7 min after addition of CCAP, and the record was taken $10 \mathrm{~min}$ after CCAP addition. At this stage, animals respond to neither EH nor ETH.

shown that neuromodulators can elicit a behavior by acting on the CNS as well as in the periphery (Kravitz, 1988). Although in Manduca cardioactive peptides are released peripherally around the time of ecdysis (Tublitz and Truman, 1985), it was not anticipated that any of these peptides (e.g., CCAP) played a role centrally in activating the ecdysis circuit. In this paper we present for the first time direct evidence that CCAP acts centrally as a neuromodulator during ecdysis. We show that CCAP activates the ecdysis motor program while terminating pre-ecdysis behavior. Consequently, CCAP release seems to be the key step in switching from the pre-ecdysis to the ecdysis motor program.

\section{Role of ETH in pre-ecdysis and ecdysis behaviors}

When injected into intact larvae or applied to the isolated whole CNS, ETH causes pre-ecdysis behavior followed by ecdysis (Zitnan et al., 1996). Our results (Fig. 4) confirm that ETH application causes the CNS to produce these two sequential motor programs. Behavioral studies indicate, however, that ETH triggers ecdysis indirectly. For example, when the larval CNS is transected before ETH injection, pre-ecdysis behavior occurs along the entire animal, but only the part of the animal anterior to the CNS cut subsequently displays ecdysis (Ewer et al., 1997). Our recordings from the isolated abdominal CNS are consistent with these findings. Although ETH application initiated the rapid onset of preecdysis motor bursts, there was no subsequent shift to ecdysis. Rather, the abdominal CNS continued generating pre-ecdysis bursts long after whole CNS preparations would have shifted into the ecdysis phase.

Our data support the observations of Zitnan et al. (1996) that peripherally released ETH directly induces pre-ecdysis. ETH evoked pre-ecdysis bursts from the abdominal CNS with the ganglionic sheath either intact or removed. Although the response of the abdominal CNS to ETH is robust, it diminishes over time. In the continuous presence of ETH the average duration of pre-ecdysis bursting was $>80 \mathrm{~min}$, but in a few preparations the pre-ecdysis bursts ceased altogether by $90 \mathrm{~min}$. The reason for this rundown is not clear. Under normal conditions, however, the ecdysis phase begins long before the pre-ecdysis response has degraded.

ETH elicited ecdysis motor output from the CNS only if the connection between the brain and ventral nerve cord was intact. This finding agrees with previous work showing that ETH excites the ventromedial neurons in the brain (Ewer et al., 1997) that then release EH throughout the CNS and into the blood (Hewes and Truman, 1991). Central EH release elevates cGMP levels in 


\section{ETH}

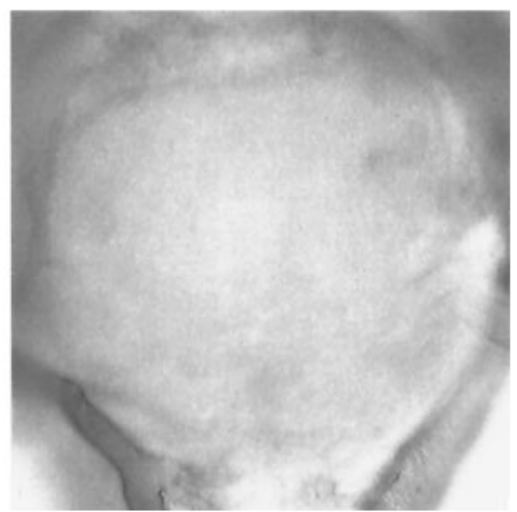

Pre-ecdysis
only
ETH

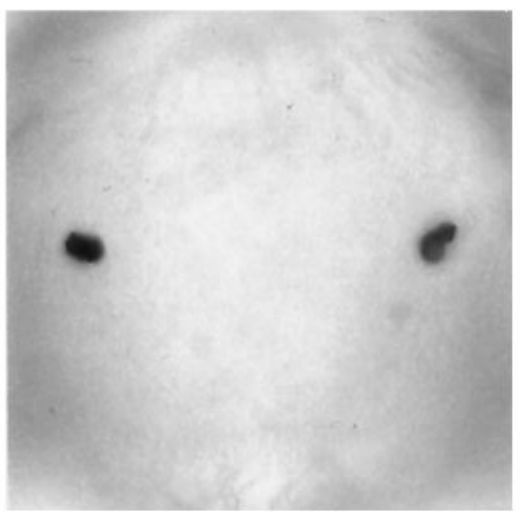

Pre-ecdysis and ecdysis
CCAP

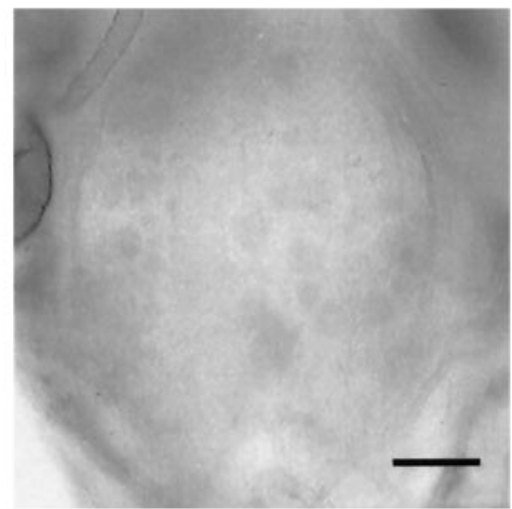

Ecdysis
only

Figure 7. Typical cGMP immunoreactivity found in the abdominal ganglia of isolated nervous systems after various peptide treatments. Left, Application of $1 \mu \mathrm{M}$ ETH to the abdominal CNS resulted only in the generation of pre-ecdysis bursts; such nervous systems showed no cells with elevated cGMP. Middle, Application of $1 \mu \mathrm{M}$ ETH to a whole CNS resulted in pre-ecdysis, followed by ecdysis motor bursts; these nervous systems consistently showed elevated levels of cGMP within the cells of the Cell $27 / 704$ group. Right, Application of $0.1 \mu \mathrm{M}$ CCAP to the abdominal CNS resulted in the ecdysis motor output; the Cell 27/704 group showed no elevated cGMP levels under these conditions. Scale bar, $50 \mu \mathrm{M}$.
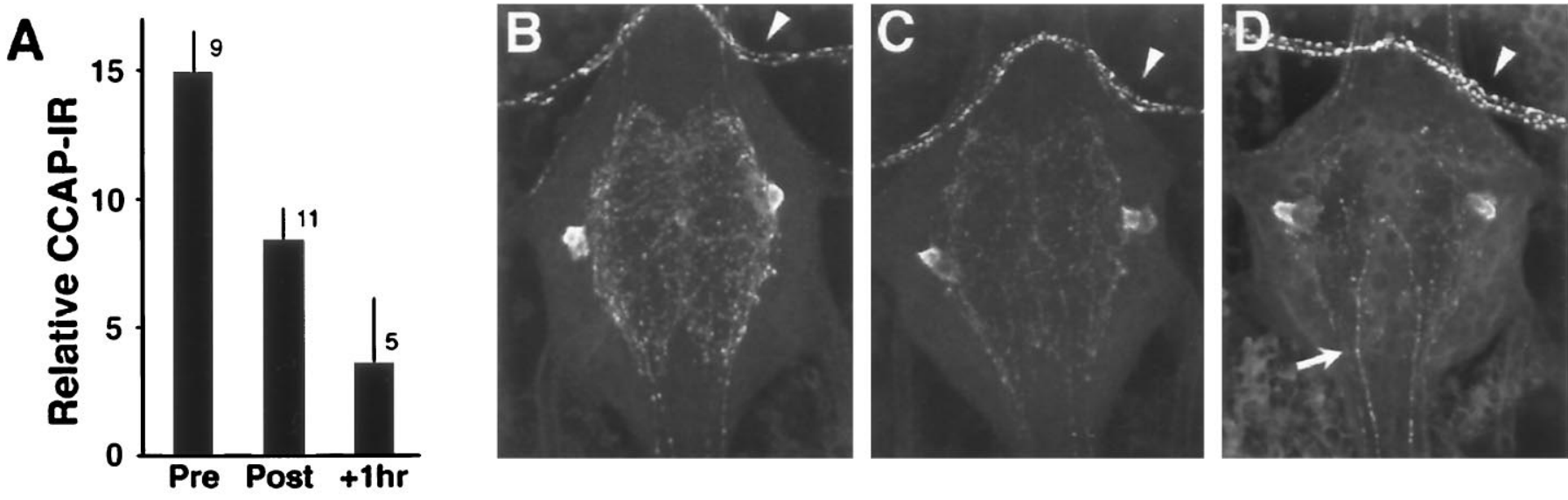

Figure 8. Confocal analysis of the changes in CCAP immunoreactivity (CCAP-IR) in the neuritic arbor of the paired Cells 27 and 704 in ganglion A6 of second instar larvae. A, Quantification of the relative amount of CCAP-IR before the start of ecdysis (Pre), immediately after a normal ecdysis (Post), and after larvae had been induced to undergo ecdysis for an additional hour because of the premature removal of a ring of old cuticle around segment A3 $(+1 h r)$. Bars show the mean + SE for the number of preparations indicated. $B-D$, Representative examples of CCAP-IR in ganglia from the three respective groups. Arrowheads indicate CCAP-IR in the transverse nerve anterior to each ganglion; this is from a peripheral neuron that does not release CCAP at ecdysis. Arrow indicates descending axons of Cell 27.

the Cell 27/704 group (Ewer et al., 1997), thereby enhancing their excitability (Gammie and Truman, 1997). We find here that the peptide released by these cells, CCAP, activates the ecdysis motor output.

\section{Role of CCAP in the generation of the ecdysis behavior}

CCAP is a peptide that is found throughout the arthropods in a highly conserved set of neurons (Dircksen, 1994). Moreover, in insects, a cGMP increase at ecdysis is a common response seen in these cells (Ewer and Truman, 1996). This study is the first demonstration that CCAP acts centrally to elicit the rhythmical ecdysis bursts. The motor bursts produced by the CNS in response to CCAP show no initial pre-ecdysis patterning, but they start with a defined ecdysis patterning (Fig. 1). In our longest experiments
(90 $\mathrm{min}$ ), the continued presence of CCAP resulted in the production of ecdysis bursts throughout the entire experiment, a duration well beyond that normally seen in intact animals. The ecdysis bursts changed only slightly through the recording period (Fig. $1 B$ ). The frequency of motor bursts did not change with different CCAP concentrations $\left(10^{-9}-10^{-6} \mathrm{M}\right)$. Additionally, because $10^{-10} \mathrm{M}$ CCAP did not elicit ecdysis motor bursts, CCAP may act as an all-or-none switch. Importantly, this effect is reversible, because the ecdysis program can be turned on and off repeatedly by the addition and withdrawal of the peptide. Thus, CCAP acts tonically, with a sustained presence required for continuous behavioral output.

The effects of CCAP are not stage specific. The abdominal CNS of intermolt larvae, when treated with CCAP, generated ecdysis 
Figure 9. Diagram showing the neuromodulator pathways controlling preecdysis and ecdysis behaviors. Release of ETH from the Inka cells both initiates pre-ecdysis and excites the ventromedial (VM) neurons that contain eclosion hormone. Because they are part of a positive feedback loop, the Inka cells and VM neurons release almost all of their peptide stores. EH release within the CNS triggers cGMP upregulation in the Cell 27/704 group, causing the central and peripheral (not shown) release of CCAP. Centrally released CCAP both activates the ecdysis motor program and terminates pre-ecdysis. Sensory input (possibly from bristle hairs deformed by the pressure of the old cuticle) may maintain excitation of the Cell 27/704 group to insure CCAP release and the continuation of ecdysis until the cuticle is shed. Removal of the cuticle eliminates the sensory input, resulting in the cessation of CCAP release and of ecdysis behavior.

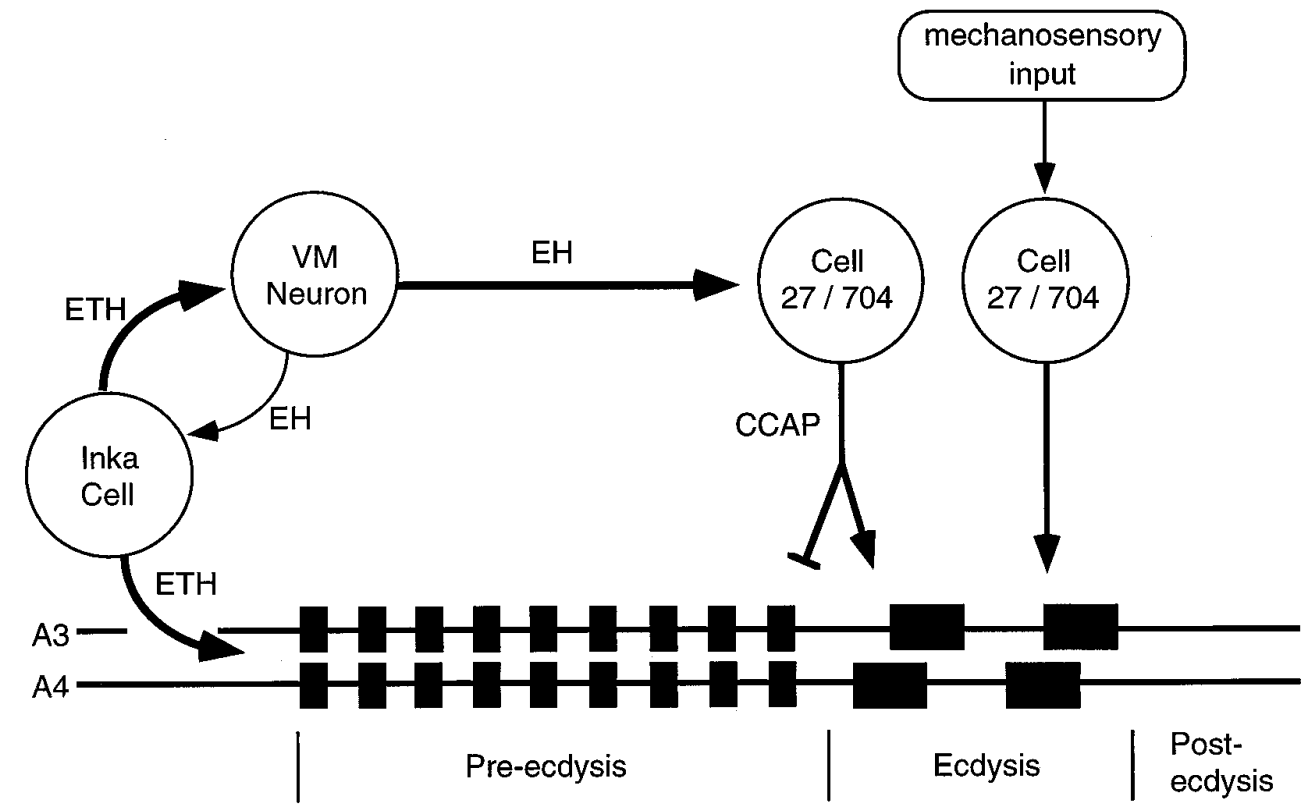

bursts identical to those produced by animals late in the molt. The lack of stage specificity for CCAP action is in marked contrast to both EH and ETH, which show relatively narrow sensitive periods for their action on the CNS (Truman et al., 1983; Zitnan et al., 1996).

Unlike ETH, which apparently can traverse the blood-brain barrier, CCAP is not effective when injected into animals or applied to the intact CNS in vitro (Fig. $1 C$ ). Thus, a central release of CCAP is required. Our immunocytochemical data support this idea. We see a marked depletion in central CCAP during the course of normal ecdysis, with the magnitude of depletion proportional to the duration of the behavior. Our experiments with girdled larvae show that CCAP continues to be released if the animals become trapped and cannot shed their cuticle. These trapped larvae eventually stop their ecdysis attempts, presumably because of the exhaustion of their CCAP stores.

Both behavioral data (Ewer et al., 1997) and the electrophysiological data presented here show an invariant association of the cGMP elevation in the Cell 27/704 group with ecdysis when the behavior is initiated by either ETH or EH. This cGMP increase, however, is not seen when CCAP is used to evoke ecdysis motor bursts from the abdominal CNS. This result is consistent with the hypothesis that cGMP upregulation is upstream of CCAP release and the induction of ecdysis.

CCAP also causes the premature termination of the pre-ecdysis motor program. ETH application to an abdominal CNS resulted in the long-lasting performance of pre-ecdysis bursts, with an average duration $>80 \mathrm{~min}$ (Fig. 2). Application of CCAP to the abdominal CNS shortly after ETH application, however, resulted in a brief period during which both pre-ecdysis and ecdysis bursts occurred together followed by only ecdysis bursts (Fig. 5). In vivo, the transition from pre-ecydsis to ecdysis may be facilitated by the normal rundown of ETH action, but in our experiments the average duration of ETH-induced pre-ecdysis bursting was significantly shortened by the presence of CCAP. Also, because ETH was unable to disrupt ecdysis bursts or trigger stable pre-ecdysis patterning when applied after CCAP, we conclude that CCAP has a dominant action on the CNS compared with ETH.

\section{Behavioral implications}

Figure 9 summarizes our current understanding of the interaction of the neuromodulators that regulate the sequential display of pre-ecdysis and ecdysis. In an intact Manduca, the pre-ecdysis behavior (which loosens the cuticle) is followed by the ecdysis behavior (which sheds the cuticle). Recordings from the isolated CNS likewise show this sequence (Fig. 4). ETH secretion is likely the normal stimulus to initiate the pre-ecdysis behavior (Fig. 3) (Zitnan et al., 1996). ETH also stimulates EH release and vice versa (Ewer et al., 1997), and this positive feedback interaction results in both EH (Ewer et al., 1997) and ETH (Zitnan et al., 1996) undergoing essentially complete release. Lower concentrations of ETH initiate pre-ecdysis before higher concentrations activate the ecdysis pathway (Zitnan et al., 1996). The ETHinduced release of $\mathrm{EH}$ then stimulates cGMP elevation in the Cell 27/704 group (Ewer et al., 1997), which increases their excitability (Gammie and Truman, 1997) and leads to CCAP release centrally. CCAP then acts in a hierarchical manner on the CNS and activates the ecdysis motor output while it terminates pre-ecdysis.

Unlike the phasic release of $\mathrm{EH}$, the immunocytochemical data on CCAP depletion along with data from intracellular recordings (Gammie and Truman, 1997) suggest that this peptide is released in a lower-level, tonic manner. Indeed, at the end of a normal ecdysis, the central arbors of the CCAP cells still retain about half of their pre-ecdysis levels of peptide (Fig. 8). This residual peptide may provide a safety factor, so that if the animal encounters difficulties in shedding the cuticle, such as in the girdled larvae, there will still be CCAP present that can be used to maintain an extended ecdysis motor pattern. We suggest that mechanosensory input stimulated by the presence of the old cuticle might impinge directly onto the CCAP cells. In Manduca, cGMP levels remain elevated in some cells for up to $3 \mathrm{hr}$ after ecdysis (Ewer et al., 1994), and we hypothesize that as long as their cGMP levels remained elevated, this sensory input would maintain the firing of the CCAP cells and hence prolong CCAP release and the consequent expression of the ecdysis motor pattern. We have no direct information from Manduca that prolonged ecdysis prolongs the upregulation of cGMP. 
Finally, the sequential production of the two motor programs is facilitated by modifications on at least three levels. First, the concentration of ETH required to elicit the pre-ecdysis motor pattern is much lower than that needed to cause ecdysis (Zitnan et al., 1996). Consequently, early low levels of ETH will start pre-ecdysis before the later high levels activate the pathway leading to ecdysis. Second, ETH release and action have already peaked by the time the Cell 27/704 group is first activated by EH (Ewer et al., 1997). Third, the response of the CNS to the two peptides is hierarchical, because CCAP turns off the behavior produced by ETH as well as turns on the second behavior in the sequence.

\section{REFERENCES}

Bell RA, Joachim FG (1976) Techniques for rearing laboratory colonies of tobacco hornworms and pink bollworms. Ann Entomol Soc Am 69:365-373.

Carlson JR (1977) The imaginal ecdysis of the cricket (Teleogryllus oceanicus). J Comp Physiol [A] 115:299-317.

Cheung CC, Loi PK, Sylwester AW, Lee TD, Tublitz NJ (1992) Primary structure of a cardioactive neuropeptide from the tobacco hawkmoth, Manduca sexta. FEBS Lett 313:165-168.

Copenhaver PF, Truman JW (1982) The role of eclosion hormone in the larval ecdyses of Manduca sexta. J Insect Physiol 28:695-701.

Davis NT, Homberg U, Dircksen H, Levine RB, Hildebrand JG (1993) Crustacean cardioactive peptide-immunoreactive neurons in the hawkmoth Manduca sexta and changes in their immunoreactivity during postembryonic development. J Comp Neurol 338:612-627.

De Vente J, Steinbusch HWM, Shipper J (1987) A new approach to immunocytochemistry of $3^{\prime}, 5^{\prime}$-cyclic guanosine monophosphate: preparation, specificity, and initial application of a new antiserum against formaldehyde-fixed $3^{\prime}, 5^{\prime}$-cyclic guanosine monophosphate. Neuroscience 22:361-373.

Dircksen H (1994) Distribution and physiology of crustacean cardioactive peptide in arthropods. In: Perspectives in comparative endocrinology (Davey KG, Peter RE, Tobe SS, eds), pp 139-148. Ottawa: National Research Council of Canada.

Ewer J, Truman JW (1996) Increases in cyclic GMP occur at ecdysis in an evolutionarily conserved insect neuronal network. J Comp Neurol 370:330-341.

Ewer J, De Vente J, Truman JW (1994) Neuropeptide induction of cyclic GMP increases in the insect CNS: resolution at the level of single identifiable neurons. J Neurosci 14:7704-7712.

Ewer J, Gammie SC, Truman JW (1997) Control of insect ecdysis by a positive-feedback endocrine system: roles of eclosion hormone and ecdysis triggering hormone. J Exp Biol 200:869-881.

Flamm RE, Harris-Warrick RM (1986) Aminergic modulation in lobster stomatogastric ganglion. II. Target neurons of dopamine, octopamine and serotonin within the pyloric circuit. J Neurophysiol 55:847-865.

Gammie SC, Truman JW (1997) An endogenous elevation of cGMP increases the excitability of identified insect neurosecretory cells. J Comp Physiol [A] 180:329-338.

Grillner S, Zangger P (1979) On the central generation of locomotion in the low spinal cat. Exp Brain Res 34:241-261.

Hewes RS, Truman JW (1991) The roles of central and peripheral eclosion hormone release in the control of ecdysis behavior in Manduca sexta. J Comp Physiol [A] 168:697-707.

Johnson BR, Peck JH, Harris-Warrick RM (1993) Amine modulation of electrical coupling in the pyloric network of the lobster stomatogastric ganglion. J Comp Physiol [A] 172:715-732.

Johnston RM, Levine RB (1996) Crawling motor patterns induced by pilocarpine in isolated larval nerve cords of Manduca sexta. J Neurophysiol 76:3178-3195.

Kravitz EA (1988) Hormonal control of behavior: amines and the biasing of behavioral output in lobsters. Science 241:1775-1781.

McClellan AD, Brown GD, Getting PA (1994) Modulation of swimming in Tritonia: excitatory and inhibitory effects of serotonin. J Comp Physiol [A] 174:257-266.

Miles CI, Weeks JC (1991) Developmental attenuation of the pre-ecdysis motor pattern in the tobacco hornworm, Manduca sexta. J Comp Physiol [A] 140:179-190.

Moulins M, Cournil I (1982) All-or-none control of the bursting properties of the pacemaker neurons of the lobster pyloric pattern generator. J Neurobiol 13:447-458.

Novicki A, Weeks JC (1995) A single pair of interneurons controls motor neuron activity during pre-ecdysis compression behavior in larval Manduca sexta. J Comp Physiol [A] 176:45-54.

Reynolds S (1980) Integration of behaviour and physiology in ecdysis. Adv Insect Physiol 15:475-595.

Simmers J, Meyrand P, Moulins M (1995) Modulation and dynamic specification of motor rhythm-generating circuits in crustacea. J Physiol (Paris) 89:195-208.

Sombati S, Hoyle G (1984) Generation of specific behaviors in a locust by local release into neuropil of the natural neuromodulator octopamine. J Neurobiol 15:481-506.

Trimmer BA, Weeks JC (1989) Effects of nicotinic and muscarinic agents on an identified mononeurone and its direct afferent inputs in larval Manduca sexta. J Exp Biol 144:303-337.

Truman JW, Rountree DB, Reiss SE, Schwartz LM (1983) Ecdysteroids regulate the release and action of eclosion hormone in the tobacco hornworm, Manduca sexta (L.). J Insect Physiol 29:895-900.

Tublitz NJ, Truman JW (1985) Insect cardioactive peptides. II. Neurohormonal control of heart activity by two cardioacceleratory peptides in the tobacco hawkmoth, Manduca sexta. J Exp Biol 114:381-395.

Weeks JC, Truman JW (1984) Neural organization of peptide-activated ecdysis behaviors during metamorphosis of Manduca sexta. J Comp Physiol [A] 155:407-422.

Zitnan D, Kingan TG, Hermesman JL, Adams ME (1996) Identification of ecdysis-triggering hormone from an epitracheal endocrine system. Science 271:88-91. 